Yuhong Long, Xiaoyu Hu and Jingwen Ran*

\title{
Crystal structure of poly[ $\left(\mu_{4}\right.$-benzene- 1,2,4,5-tetracarboxylato)-bis $\left(\mu_{2}\right.$-adipohydrazide) dicadmium], $\mathrm{C}_{11} \mathrm{H}_{15} \mathrm{~N}_{4} \mathrm{O}_{6} \mathrm{Cd}$
}

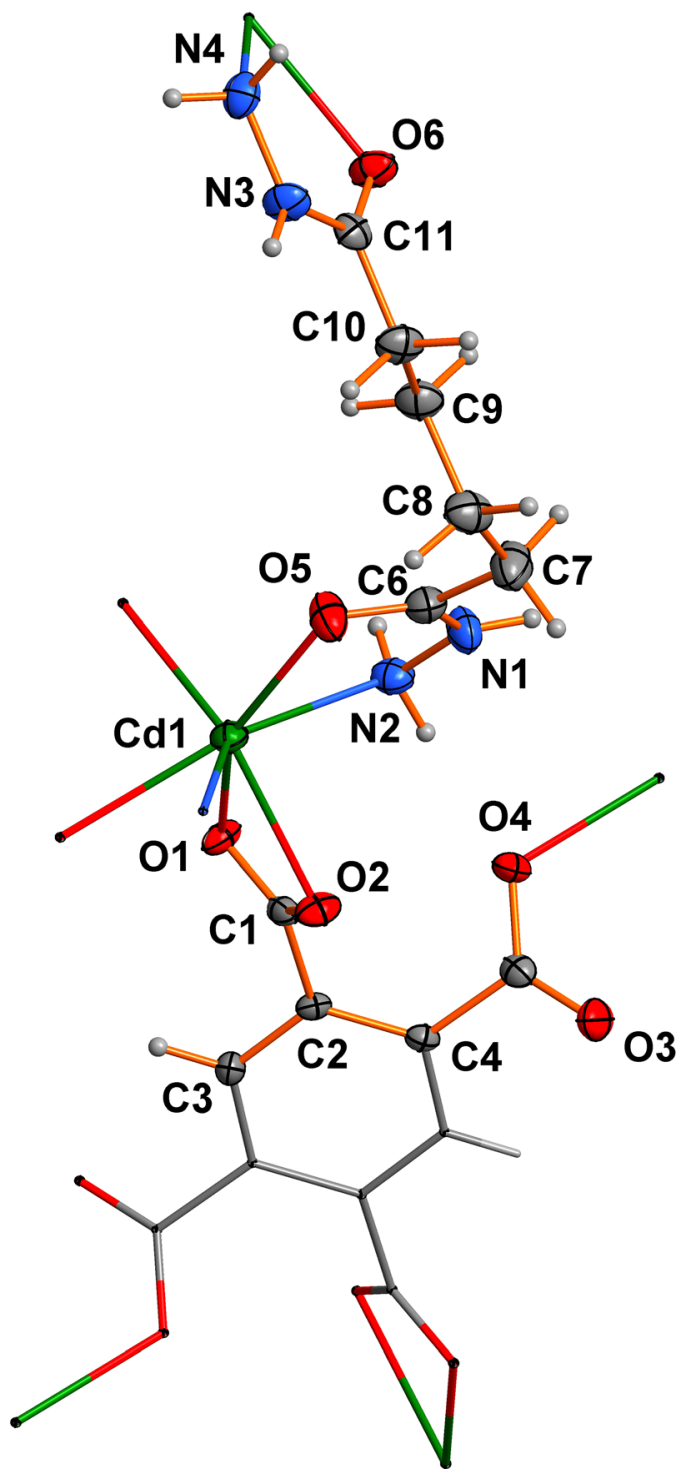

*Corresponding author: Jingwen Ran, Hubei Key Laboratory for Processing and Application of Catalytic Materials, College of Chemistry and Chemical Engineering, Huanggang Normal University, Huanggang 438000, China, E-mail: ranjingwen@hgnu.edu.cn. https://orcid.org/0000-0001-8701-6192

Yuhong Long and Xiaoyu Hu, Hubei Key Laboratory for Processing and Application of Catalytic Materials, College of Chemistry and Chemical Engineering, Huanggang Normal University, Huanggang 438000, China https://doi.org/10.1515/ncrs-2021-0309

Received July 29, 2021; accepted August 29, 2021;

published online September 8, 2021

\begin{abstract}
$\mathrm{C}_{11} \mathrm{H}_{15} \mathrm{CdN}_{4} \mathrm{O}_{6}$, monoclinic, $P 2_{1} / n$ (no. 14), $a=11.1387(15) \AA$, $b=10.3933(14) \AA, c=12.2891(17) \AA, \beta=105.319(2)^{\circ}, Z=4$, $V=1372.1(3) \AA^{3}, R_{\mathrm{gt}}(F)=0.0286, w R_{\mathrm{ref}}\left(F^{2}\right)=0.0477$, $T=296(2) \mathrm{K}$.
\end{abstract}

CCDC no.: 2106207

Table 1 contains crystallographic data and Table 2 contains the list of the atoms including atomic coordinates and displacement parameters.

\section{Source of material}

The reagents were purchased from commercial sources and used without further purification.

A mixture of benzene-1,2,4,5-tetracarboxylic acid (0.25 mmol, $63.5 \mathrm{mg}), \mathrm{NaOH}(1.0 \mathrm{mmol}, 40 \mathrm{mg}$ ) and deionized water $(5 \mathrm{~mL})$ was placed in a high-pressure resistant glass bottle $(10 \mathrm{~mL})$, the bottle was sealed and heated at $353 \mathrm{~K}$ for $30 \mathrm{~min}$. After that, cadmium acetate dihydrate $(0.5 \mathrm{mmol}, 133 \mathrm{mg})$ and adipohydrazide ( $0.5 \mathrm{mmol}, 87 \mathrm{mg}$ ) were added, and the bottle was sealed and heated at $393 \mathrm{~K}$ for $24 \mathrm{~h}$ and cooled to room temperature. Colorless prism crystals were obtained by filtration, washed with deionized water and dried in air. Yield $46 \%$ based on $\mathrm{Cd}(\mathrm{II})$.

\section{Experimental details}

$\mathrm{H}$ atoms were added using riding models. Their $U_{\text {iso }}$ values were set to $1.2 U_{\text {eq }}$ of the parent atoms.

\section{Comment}

Researchers have focused on coordination polymers due to their versatile architecture and potential applications for 
Table 1: Data collection and handling.

\begin{tabular}{ll}
\hline Crystal: & Colorless prism \\
Size: & $0.32 \times 0.31 \times 0.28 \mathrm{~mm}$ \\
Wavelength: & Mo $K \alpha$ radiation $(0.71073 \AA)$ \\
$\mu:$ & $1.63 \mathrm{~mm}^{-1}$ \\
Diffractometer, scan mode: & Bruker Apex-III, $\varphi$ and $\omega$ \\
$\theta_{\text {max }}$, completeness: & $27.0^{\circ}, 99 \%$ \\
$N\left(h k l_{\text {measured }}, N(h k l)_{\text {unique }}, R_{\text {int }}:\right.$ & $7847,2966,0.035$ \\
Criterion for $I_{\text {obs }}, N(h k l)_{\text {gt }}:$ & $I_{\text {obs }}>2 \sigma\left(I_{\text {obs }}\right), 2324$ \\
$N(\text { param })_{\text {refined }}:$ & 200 \\
Programs: & Bruker [1], SheLx [2, 3], DiAmOND [4] \\
\hline
\end{tabular}

Table 2: Fractional atomic coordinates and isotropic or equivalent isotropic displacement parameters $\left(\AA^{2}\right)$.

\begin{tabular}{|c|c|c|c|c|}
\hline Atom & $x$ & $y$ & $z$ & $U_{\text {iso }}{ }^{*} / U_{\text {eq }}$ \\
\hline Cd1 & $0.60643(2)$ & $0.19229(2)$ & $0.16712(2)$ & $0.01766(8)$ \\
\hline 01 & $0.82720(17)$ & $0.1852(2)$ & $0.16547(16)$ & 0.0218 \\
\hline 02 & 0.69441 (17) & $0.0556(2)$ & 0.04887 (16) & $0.0222(5)$ \\
\hline 03 & 0.82900 (19) & $-0.2851(2)$ & $0.04777(18)$ & $0.0282(5)$ \\
\hline 04 & 0.85391 (19) & -0.15641 (19) & $0.19838(17)$ & $0.0254(5)$ \\
\hline 05 & 0.43704 (19) & $0.0986(2)$ & 0.22782 (19) & $0.0316(6)$ \\
\hline 06 & 0.12613 (19) & $0.1328(2)$ & 0.54508 (17) & $0.0288(5)$ \\
\hline N1 & $0.5739(2)$ & $-0.0581(3)$ & $0.3011(2)$ & $0.0290(7)$ \\
\hline $\mathrm{H} 1$ & 0.586555 & -0.133593 & 0.330668 & $0.035^{\star}$ \\
\hline N2 & $0.6746(2)$ & $0.0170(2)$ & $0.2884(2)$ & $0.0251(6)$ \\
\hline $\mathrm{H} 2 \mathrm{~A}$ & 0.725321 & -0.031738 & .260864 & $0.030^{\star}$ \\
\hline $2 B$ & 0.717310 & .045954 & 0.355689 & $0.030^{*}$ \\
\hline N3 & $-0.0373(2)$ & $0.2228(3)$ & $0.4215(2)$ & $0.0279(7)$ \\
\hline $\mathrm{H} 3 \mathrm{~A}$ & -0.081536 & 9573 & 2884 & $0.033^{*}$ \\
\hline N4 & $-0.0665(2)$ & $0.2965(3)$ & $0.5080(2)$ & $0.0269(6)$ \\
\hline H4A & -0.130412 & .260780 & .527426 & $0.032^{\star}$ \\
\hline H4B & -0.088622 & 0.375532 & 0.482705 & $0.032^{\star}$ \\
\hline $\mathrm{C} 1$ & $0.8026(3)$ & $0.0981(3)$ & $0.0910(2)$ & $0.0172(7)$ \\
\hline$C 2$ & $0.9070(2)$ & $0.0450(3)$ & $78(2)$ & $0.0137(6)$ \\
\hline C3 & $0.9776(3)$ & $0.1300(3)$ & $0.0027(2)$ & $0.0161(6)$ \\
\hline H3 & 0.962917 & 0.217875 & .005232 & $0.019^{\star}$ \\
\hline $\mathrm{C} 4$ & $0.9307(2)$ & $-0.0869(3)$ & $0.0459(2)$ & $0.0137(6)$ \\
\hline C5 & $0.8648(2)$ & $-0.1853(3)$ & $0.1000(2)$ & $0.0185(6)$ \\
\hline C6 & $0.4586(3)$ & $-0.0109(3)$ & $0.2668(3)$ & $0.0251(7)$ \\
\hline C7 & $0.3576(3)$ & $-0.1045(3)$ & $0.2747(3)$ & $0.0388(9)$ \\
\hline H7A & 0.383275 & -0.147862 & 0.347062 & $0.047^{\star}$ \\
\hline H7B & 0.350671 & -0.169223 & 16551 & $0.047^{\star}$ \\
\hline C8 & 0.2300 & $-0.0464(3)$ & $0.2629(3)$ & $0.0322(9)$ \\
\hline $\mathrm{H} 8 \mathrm{~A}$ & 0.209268 & 0.007089 & 0.195782 & $0.039 *$ \\
\hline H8B & 0.169497 & -0.115533 & 0.251406 & $0.039^{\star}$ \\
\hline $\mathrm{C} 9$ & $0.2176(3)$ & 0.0338 & $0.3620(3)$ & $0.0288(8)$ \\
\hline $\mathrm{H} 9 \mathrm{~A}$ & 0.264359 & 0.112985 & 0.364786 & $0.035^{\star}$ \\
\hline H9B & 0.252117 & -0.013060 & 0.431484 & $0.035^{\star}$ \\
\hline C10 & 0.0814 (3) & $0.0653(3)$ & $0.3518(3)$ & $0.0274(8)$ \\
\hline $\mathrm{H} 10 \mathrm{~A}$ & 0.048202 & 0.112498 & 0.282297 & $0.033^{\star}$ \\
\hline $\mathrm{H} 10 \mathrm{~B}$ & 0.035221 & -0.014663 & 0.346518 & $0.033^{*}$ \\
\hline C11 & 0.0599 (3) & $0.1426(3)$ & $0.4480(3)$ & $0.0205(7)$ \\
\hline
\end{tabular}

luminescent properties, magnetic interactions, catalytic ability, and so on $[5,6]$. As a versatile ligand benzene1,2,4,5-tetracarboxylate has been widely used to construct transition metal or rare earth metal complexes using its multiple coordination function modes.

The asymmetric unit of the complex consists of one $\mathrm{Cd}$ ion, one adipohydrazide ligand and one half of a fully deprotonated benzene-1,2,4,5-tetracarboxylate $\left(\mathrm{btc}^{4-}\right)$. The $\mathrm{btc}^{4-}$ ligand is located on a center of inversion (see the Figure). The central $\mathrm{Cd}$ ion is coordinated by a chelating carboxylate group (01 and $\mathrm{O} 2)$ and a monodentate carboxylate group (04) from two $\mathrm{btc}^{4-}$ ligands. In addition, two adipohydrazide ligands provide two oxygen atoms ( 05 and O6) and two nitrogen atoms (N2 and N4) to coordinate with the central cadmium ion, forming a distorted single capped octahedron. Each Cd ion is connected by two btc ${ }^{4-}$ ligands to form a two-dimensional network with a rhombic-shaped grid [7]. The adipohydrazide ligands are interspersed in the twodimensional structure, ensuring the $\mathrm{Cd}$ ion to form a sevencoordinated conformation, enhancing the stability of the coordination polymer. The $\mathrm{Cd}-\mathrm{O}$ bond distances fall in the range of 2.239(2)-2.4646 (19) $\AA$ and the $\mathrm{Cd}-\mathrm{N}$ bond distances are $2.349(2)$ and 2.356(2) $\AA$. All the bond lengths around the $\mathrm{Cd} 1$ center are within the normal range $[8,9]$. The $\mathrm{N}-\mathrm{N}$ bond distances are 1.410(3) and 1.415(3) $\AA$, which are in good agreement with the reported information $[10,11]$.

Author contributions: All the authors have accepted responsibility for the entire content of this submitted manuscript and approved submission.

Research funding: This work was supported by Hubei Provincial Central Committee Guiding Local Science and Technology Development Project (No. 2019ZYYD074).

Conflict of interest statement: The authors declare no conflicts of interest regarding this article.

\section{References}

1. Bruker. APEX2, SAINT and SADABS; Bruker AXS Inc.: Madison, WI, USA, 2009.

2. Sheldrick G. M. SHELXTL - integrated space-group and crystalstructure determination. Acta Crystallogr. 2015, A71, 3-8.

3. Sheldrick G. M. Crystal structure refinement with SHELXL. Acta Crystallogr. 2015, C71, 3-8.

4. Brandenburg K. DIAMOND. Visual Crystal Structure Information System. Ver. 4.0; Crystal Impact: Bonn, Germany, 2015.

5. Zhou Y., Yoon J. Recent progress in fluorescent and colorimetric chemosensors for detection of amino acids. Chem. Soc. Rev. 2012, $41,52-67$. 
6. Dhakshinamoorthy A., Garcia H. Catalysis by metal nanoparticles embedded on metal-organic frameworks. Chem. Soc. Rev. 2012, 41, 5262-5284.

7. Shi R., Zhu X., Lin S. Y., Su Y. Y., Zhang S. Y. Crystal structure of poly [aqua- $\left(\mu_{4}\right.$-benzene-1,2,4,5-tetracarboxylato-bis $\left[\mu_{2}-1(1 H\right.$-imidazol1-yl)benzyl)-1H-1,2,4-triazol-dinickel(II)], $\mathrm{NiC}_{17} \mathrm{H}_{14} \mathrm{~N}_{5}$. Z. Kristallogr. N. Cryst. Struct. 2020, 235, 817-819.

8. Cai H., Xu C., Zhou Y. P., Tong X. Q., Guo Y. Molecular tectonics of mixed-ligand metal-organic frameworks: positional isomeric effect, and structural diversification. J. Mol. Struct. 2016, 1108, 263-268.
9. Lin J. D., Cheng J. W., Du S. W. Five $d_{10}$ 3D metal-organic frameworks constructed from aromatic polycarboxylate acids and flexible imidazole-based ligands. Cryst. Growth Des. 2008, 8, 3345-3353.

10. Lo K. M., Lee S. M., Tiekink E. R. T. Crystal structure of heanedihydrazide, $\mathrm{C}_{6} \mathrm{H}_{14} \mathrm{~N}_{4} \mathrm{O}_{2}$. Z. Kristallogr. N. Cryst. Struct. 2020, 235, 1257-1258.

11. Zabierowski P., Oszajca M., Hodorowicz M., Matoga D. Ladderchain zinc coordination polymers based on adipic acid dihydrazide precursor: synthesis and structural transformations. Polyhedron 2017, 121, 25-36. 